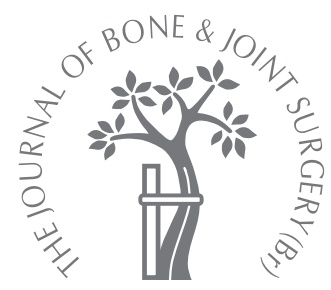

\title{
Long-term survival of concurrent meniscus allograft transplantation and repair of the articular cartilage
}

\author{
A PROSPECTIVE TWO- TO 12-YEAR FOLLOW-UP REPORT
}

K. R. Stone, W. S. Adelson, J. R. Pelsis, A. W. Walgenbach, T. J. Turek

From The Stone

Research

Foundation, San

Francisco, California, United States

K. R. Stone, MD, Orthopaedic Surgeon

A. W. Walgenbach, MSN, RNNP, Nurse Practitioner The Stone Clinic

W. S. Adelson, MS, Senior Research Co-ordinator, Staff Scientist

J. R. Pelsis, MHS, Research Associate

T. J. Turek, BS, Staff Scientist Stone Research Foundation 3727 Buchanan Street, San Francisco, California 94123, USA.

Correspondence should be sent to Dr K. R. Stone; e-mail: kstonemd@stoneclinic.com

(C)2010 British Editorial Society of Bone and Joint Surgery doi:10.1302/0301-620X.92B7. $23182 \$ 2.00$

$J$ Bone Joint Surg [Br] 2010;92-B:941-8. Received 30 July 2009; Accepted after revision 11 March 2010

We describe 119 meniscal allograft transplantations performed concurrently with articular cartilage repair in 115 patients with severe articular cartilage damage. In all, $53(46.1 \%)$ of the patients were over the age of 50 at the time of surgery. The mean follow-up was for $\mathbf{5 . 8}$ years ( 2 months to 12.3 years), with 25 procedures $(20.1 \%$ ) failing at a mean of 4.6 years ( 2 months to $\mathbf{1 0 . 4}$ years). Of these, 18 progressed to knee replacement at a mean of 5.1 years (1.3 to 10.4). The Kaplan-Meier estimated mean survival time for the whole series was 9.9 years (SD 0.4). Cox's proportional hazards model was used to assess the effect of covariates on survival, with age at the time of surgery $(p=0.026)$ and number of previous operations $(p$ $=0.006$ ) found to be significant.

The survival of the transplant was not affected by gender, the severity of cartilage damage, axial alignment, the degree of narrowing of the joint space or medial versus lateral allograft transplantation. Patients experienced significant improvements at all periods of follow-up in subjective outcome measures of pain, activity and function (all p-values $<0.05$ ), with the exception of the seven-year Tegner index score $(p=0.076)$.

Loss of the meniscus generates increased forces on the articular cartilage of the knee and other joint structures and increases the risk of the development of arthritis., ${ }^{1,2}$ The appropriate treatment for loss of the meniscus with unicompartmental arthritis remains controversial, 3,4 with treatments including osteotomy, unicompartmental (UKR) or total knee replacement (TKR). Biological treatment, such as meniscal allograft transplantation and articular cartilage repair, might potentially slow the progression of arthritis without limiting the option for arthroplasty in the future.

Recent studies show promising outcomes for biological repair of the arthritic knee. ${ }^{5-10}$ Rue et $\mathrm{al}^{5}$ reported on 31 combined procedures of meniscal allograft transplantation and cartilage restoration with a minimum two-year follow-up and an overall success rate of $93 \%$ at a mean of 3.1 years. Farr et $\mathrm{al}^{7}$ described 29 combined meniscal allograft transplantation and autologous chondrocyte implantation with an overall success rate of $86 \%$ at a mean of 4.5 years. Cole et $\mathrm{al}^{11}$ reported on 44 meniscal allograft transplantations, 19 of which were done in combination with repair of the articular cartilage, with a success rate of $84 \%$ at a mean of 2.8 years. Verdonk et $\mathrm{al}^{12}$ described 100 meniscal allograft transplantations at a mean follow-up of 7.2 years, with seven patients receiving concomitant treatment of the articular cartilage. The cumulative survival time for both medial and lateral meniscal allografts was 11.6 years.

Our group has previously reported the twoto seven-year follow-up of meniscal allograft transplantation in patients with moderate to severe arthritis. ${ }^{8}$ We now describe a long-term investigation of concurrent meniscal allograft transplantation and articular cartilage repair in patients with severe articular cartilage damage in a larger cohort of patients with a mean follow-up approaching the longest to date.

\section{Patients and Methods}

The study was approved by an independent Institutional Review Board. Consecutive patients receiving meniscal allograft surgery between March 1997 and March 2008 were followed according to a standard protocol and as needed in the event of symptoms. Inclusion criteria included pain in the knee with irreparable damage to the meniscus, where a complex tear involved the body of the meniscus for which suture repair was not possible, or loss of more than $50 \%$ of the meniscus Outerbridge ${ }^{13}$ grade III or IV changes in the respective compartment; and range of movement of the knee of at least $90^{\circ}$. Radiological assessment of joint space narrowing was determined using the 
worst of postero-anterior flexion or anteroposterior standing views, and axial alignment by standing long-leg views. Narrowing of the joint space was evaluated pre-operatively using a modified Kellgren-Lawrence system ${ }^{14}$ simplified into three grades: normal $(\geq 5 \mathrm{~mm})$, mild to moderate $(<5 \mathrm{~mm},>1 \mathrm{~mm})$, and severe $(\leq 1 \mathrm{~mm})$ based on the height of the joint space. Damage to the articular cartilage was graded during surgery using the Outerbridge classification scale. ${ }^{13}$ Severe axial malalignment $\left(>7^{\circ}\right)$ or ligamentous instability was addressed before or at the time of meniscus allograft transplantation. Allograft sizing was based on donor-recipient sex, height, and weight which has been shown to be as accurate as radiologic sizing. ${ }^{15,16}$

Surgical technique. The allograft transplantations were performed by a single surgeon (KRS) and the same surgical team. Standard anterior, medial and lateral arthroscopic portals were used. An arthroscopic shaver was used to debride the joint, removing damaged articular cartilage, impinging osteophytes and arthrofibrotic tissues to increase joint volume and mobility. The suprapatellar pouch and patellar tendon tibial intervals were opened with the shaver if scarred. Prior to 2003, at the time of meniscal allograft transplantation medial opening wedge osteotomies $(n=15)$ were performed using a resorbable osteotomy wedge (Bionx, Blue Bell, Pennsylvania) for correction of varus malalignment ranging between $5^{\circ}$ and $14^{\circ}$. At the discretion of the senior author (KRS), meniscal allograft transplantation procedures were performed without osteotomies after this. Anterior cruciate ligament reconstruction was carried out when indicated. Medial meniscal allograft transplantation was performed arthroscopically by the threetunnel technique, ${ }^{17}$ using periosteum, but not bone blocks, at the meniscal horns. The three-tunnel technique secures the meniscus at both horns and at the posterior quarter of the meniscus in an effort to reproduce the meniscal tissue ligaments. Lateral meniscal allograft transplantation was also performed arthroscopically by the three-tunnel technique, preserving the bony block between the horns and inserting it into a bone trough. The posterior corner was secured posterior to the popliteus tendon by the three-tunnel technique.

The management of the articular cartilage was dependent on the location of the defect and the degree of degradation. Microfracture was used to treat Outerbridge grade III and IV defects if the area was $\leq 25 \mathrm{~mm}^{2}$, if it was located too far posteriorly (beyond the reach of the microfracture awl), or if it was directly under the meniscal allograft transplant on the tibial side. Articular cartilage paste grafting ${ }^{6}$ was used to treat Outerbridge grade IV defects $>25 \mathrm{~mm}^{2}$ that were accessible. This procedure includes morsellising the exposed bone area, harvesting a $8 \mathrm{~mm} \times 15 \mathrm{~mm}$ plug of articular cartilage and cancellous bone from the intercondylar notch, smashing the plug into a paste and impacting the paste into the morsellised area. The paste graft has been shown to contain progenitor cells capable of regenerating cartilage. Grade III lesions were treated with microfracture alone.
An example of the population treated in this series is presented in Figure 1.

Post-operative rehabilitation. All patients were discharged from hospital on the day of surgery with a knee brace to limit range of movement to between $0^{\circ}$ and $90^{\circ}$. They returned the next day to commence a rehabilitation programme, starting with soft-tissue mobilisation, non-operative leg bicycling, and range-of-movement exercises. Aerobic exercise was initiated early based on the positive effects of whole-body exercise on wound healing. ${ }^{18,19}$ Full weight-bearing was introduced at four weeks, based on Wolff's ${ }^{20}$ concept of bone functional adaptation to mechanical loading in a moderate protective phase until the 12th week.

Outcome assessment. All patients who were treated are included in the survival analysis. Those who completed their two-year follow-up are included in the analysis of patient-reported subjective outcomes. ${ }^{7,11}$ Failure of the allograft transplantation was defined as removal of the allograft without revision, or progression to a total or unicompartmental knee replacement. ${ }^{7,21}$ In patients in whom the primary meniscal allograft was removed and revised with implantation of a new meniscal allograft, this was noted in subsequent surgical procedures.

Patients underwent clinical examination and patientreported subjective outcomes pre-operatively and at two, three, five, seven and ten years after allograft transplantation. The International Knee Documentation Committee (IKDC) Subjective Knee Evaluation Form, ${ }^{22,23}$ Western Ontario and McMasters Osteoarthritis Index (WOMAC) ${ }^{24}$ and Tegner $^{25}$ scoring methods were used to assess pain, activity and function during follow-up. The IKDC forms were scored using the method of Anderson et al ${ }^{22}$ to control for over- and underestimation of missing values. The question in the WOMAC score asking patients to rate the level of pain experienced in the previous 48 hours for the operated knee was evaluated as an isolated assessment of pain ( $0=$ none, $1=$ mild, $2=$ moderate, $3=$ severe, $4=$ extreme $)$. The patient's highest pre-injury Tegner level was recorded and used as the denominator to create a ratio of the activity level regained at each follow-up interval (Tegner index). ${ }^{26}$ This method normalises return to activity across a diverse population. Pre-operative Tegner index ratios $>1.0$ were considered outliers and were discarded. All study data were recorded on standardised forms and verified by the current study co-ordinator using the original documents. Subjective outcome questionnaires were filled out independently of the senior author and the research team. Because few follow-up examinations occurred on the precise scheduled date of follow-up, periods were created to group subjective outcome assessments at the midpoint of each scheduled follow-up, with the exception of the baseline and two-year cut-offs. Pre-operative assessments were only included if they were completed within six months of the allograft transplantation. For the two-year follow-up, assessments ranging from 18 to 30 months post-operatively (1.5 to 2.5 years) were included. 


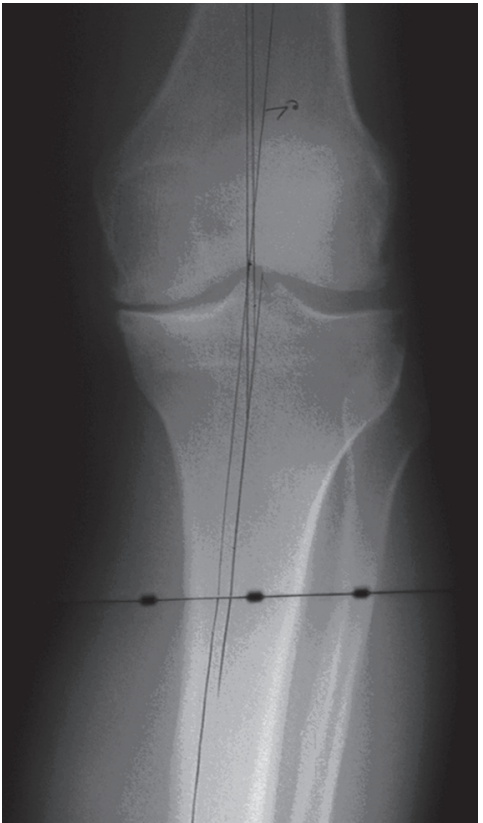

Fig. 1a

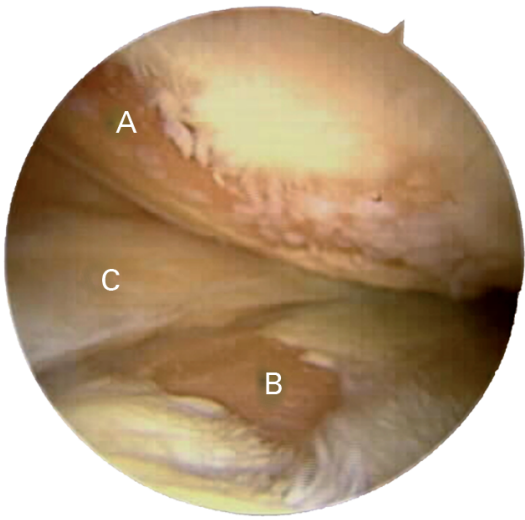

Fig. 1b

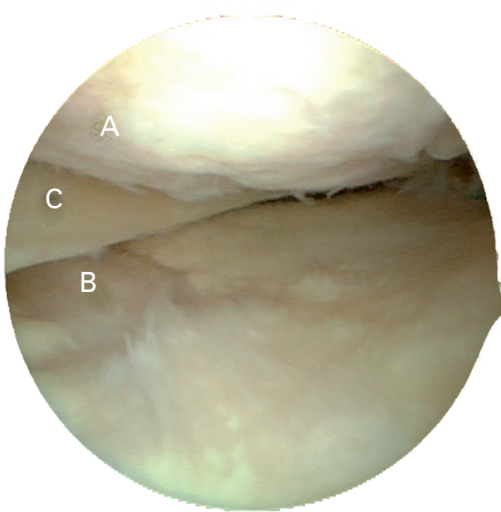

Fig. 1c

Figure 1a - Pre-operative anteroposterior long-leg weight-bearing radiograph of a 57-year-old man with a 21 -year history of symptoms since an injury while playing football. There is moderate medial joint space narrowing and varus malalignment $\left(5^{\circ}\right.$ to $\left.7^{\circ}\right)$. Figure $1 \mathrm{~b}-\operatorname{lntra}$-operative photograph of the same patient revealing Outerbridge grade IV chondral lesions of (A) the femoral condyle and (B) the tibial plateau, and (C) a severely damaged medial meniscus. Figure $1 \mathrm{c}-$ Second-look arthroscopy 3.5 years after meniscus allograft transplantation and articular cartilage repair showing reestablishment of the articular cartilage on both (A) the femoral and (B) the tibial chondral lesions, as well as (C) a healthy, integrated meniscus.

Statistical methods. Overall patient survival was analysed by the Kaplan-Meier method with $95 \%$ confidence intervals (CI). Multivariate analysis using Cox's proportional hazards model was carried out to assess the effect of covariates on allograft survival. Secondary analysis of patient-reported subjective outcome data was accomplished using Wilcoxon's rank-sum test for two independent non-parametric samples. The level of significance was set at $\mathrm{p}<0.05$. Results are presented as means and SD.

\section{Results}

Between March 1997 and March 2008, 151 meniscal allograft transplantations were performed. Patients with Outerbridge grade 0 to II articular cartilage changes $(\mathrm{n}=$ 19) and those with simultaneous medial and lateral meniscal allograft transplantations $(n=2)$ did not meet the study inclusion criteria. In all, 119 primary meniscal transplantations in 115 patients were included. Staged bilateral meniscal transplantation was undertaken in four patients. The minimum follow-up of two years was obtained in 100 transplants in 98 patients, which were included in the analysis of patient-reported subjective outcome data. The mean follow-up was for 5.8 years ( 2 months to 12.3 years). The transplantation was performed medially in 85 knees $(71.4 \%)$ and laterally in $34(28.6 \%)$. It was undertaken in $83(69.7 \%)$ male knees and $32(30.3 \%)$ female knees. The mean age at the time of surgery was 46.9 years (14.1 to $73.2)$, with $53(46.1 \%)$ patients over the age of 50 . A total of 13 patients were lost to follow-up and were censored in the survival analysis at the first follow-up visit for which no data were reported. One patient died before the study ended, with the meniscal allograft intact, and was censored accordingly.

Of the 119 transplants, 118 were associated with chronic injuries, defined as a period of three or more months from the time of injury to surgery. The mean time from the injury to surgery was 14.2 years ( 2 months to 39.7 years), with a mean of 2.1 procedures ( 0 to 9 ) performed on the affected knee prior to meniscal allograft transplantation. During $118(99.2 \%)$ of the allograft transplantations at least one additional procedure was performed, with a mean of five concomitant procedures (one to nine). These included any combination of meniscal allograft transplantation, articular cartilage paste grafting ${ }^{6}(n=67)$, microfracture $(n=69)$, medial opening tibial osteotomy $(\mathrm{n}=15)$, and anterior cruciate ligament reconstruction with a bone-patellar tendonbone allograft in ten knees, a middle-third patellar tendon autograft in six and a tendo Achillis allograft in one. Student's $t$-test revealed there was no significant difference in the mean number of concomitant procedures between those cases that failed (5.3, SD 1.6) and those that did not (4.9, SD 1.7) ( $\mathrm{p}=0.333)$.

Meniscal allograft tissue was either fresh-frozen $(\mathrm{n}=94)$, cryopreserved $(n=24)$ or irradiated $(n=1)$. The intraoperative Outerbridge classification was grade III in 22 knees $(18.5 \%)$ and grade IV in 97 knees $(81.5 \%)$. No joint space narrowing was found in 31 knees $(26.1 \%)$ at presentation, $48(40.3 \%)$ presented with mild to moderate narrowing and 


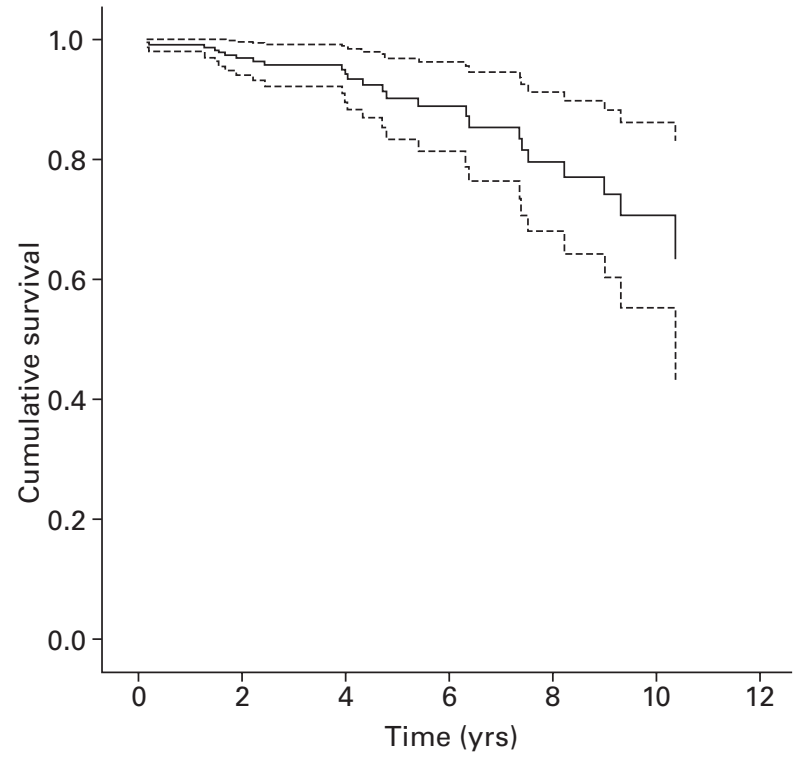

Fig. 2

Graph showing the mean Kaplan-Meier survival distribution with mean predicted survival time of 9.9 years ( 2 months to 12.4 years), ( $n=119)$, with $95 \%$ confidence intervals.

$40(33.6 \%)$ presented with severe narrowing. Neutral alignment of $<5^{\circ}$ deviation from the physiological axis was found in $73 \mathrm{knees}(58.8 \%)$ at presentation, $39(32.8 \%)$ presented with moderate malalignment $\left(5^{\circ}\right.$ to $\left.7^{\circ}\right)$, and ten $(8.4 \%)$ with severe malalignment $\left(>7^{\circ}\right)$. Of the 49 knees $(41.2 \%)$ that were malaligned at presentation, 41 were in varus $(83.7 \%)$, and eight were in valgus $(16.3 \%)$.

Subsequent procedures. A total of 56 knees $(47.0 \%)$ required up to five subsequent operations after the original meniscal allograft transplantation. Procedures included debridement $(\mathrm{n}=73)$, chondroplasty $(\mathrm{n}=39)$, notchplasty $(\mathrm{n}=$ $16)$, microfracture $(n=8)$, removal of osteophytes $(n=12)$, removal of loose bodies $(\mathrm{n}=11)$, articular cartilage paste grafting $(n=7)$, excision of a Baker's cyst $(n=2)$ and anterior cruciate ligament reconstruction $(n=1)$, many of which were performed concomitantly. Subsequent procedures directly addressing the meniscal allograft transplant are described below.

Partial meniscectomy was carried out in 23 knees $(19.3 \%), 14$ medial and nine lateral. Two patients underwent two separate operations for partial meniscectomy. In seven of the knees that underwent subsequent partial meniscectomy the meniscal transplant ultimately failed.

Repair of allograft was carried out to preserve it when judged appropriate. A total of 11 knees (9.2\%), six medial and five lateral, underwent repair surgery, one lateral meniscal allograft being repaired twice. Three cases involved suture repair of a tear of the allograft and eight involved re-attachment, with five menisci which underwent subsequent repair ultimately failing.
Revision meniscal allograft transplantation was carried out in eight knees $(6.7 \%)$ with seven being revised once and one revised twice. Revision was performed in six knees in the early post-operative period at a mean of 7 months (1.9 to 12.4). Three patients undergoing early post-operative revision had not followed the rehabilitation protocol, and one patient felt the allograft tear while turning over in bed two months post-operatively. Two cases were revised more than three years after the primary operation, one at 4.0 years, and one at 3.3 years, and then again 2.5 months later. The knee that required two revisions had an intact meniscus at the end of the study, 7.6 years after the primary operation. Two revision cases ultimately failed, with one undergoing removal of the allograft at 1.7 years and one progressing to joint replacement at 3.9 years. One revised patient died with the revision meniscus intact 4.7 years after index surgery. The remaining five revision cases are included as non-failures at a mean of 2.5 years (1.0 to 4.4 ). Complications. Early post-operative infection occurred in four knees. Three infections were deep and one was superficial. The superficial case cultured Staphylococcus epidermidis, but no organisms were seen in the synovial fluid. One of the three deep infections cultured Staphylococcus aureus, but no organisms were isolated in the others. All were treated arthroscopically with irrigation and debridement and intravenous antibiotics. All infections resolved, but one deeply infected knee ultimately suffered failure of the allograft, which was removed 12.5 months later.

Survival analysis. Kaplan-Meier survival analysis revealed an overall mean survival of the allograft of 9.9 years (SD 0.4 , 95\% CI 9.1 to 10.7 ) (Fig. 2). Because less than $50 \%$ survival was not reached, median survival estimates are not given.

Cox's proportional hazards model was used to test the confounding effects of several clinically significant covariates among allograft patients simultaneously, recognising that the hazard ratio is simply an indication of the likelihood of which group will fail first, but gives no measure of the difference in time. ${ }^{27}$ Table I shows the hazard ratios of the variables tested and their significance, as well as $95 \%$ CIs. In all, 25 (20.1\%) of the 119 meniscal allograft transplantations failed. Seven failed before two years and 18 after (Table II) with a mean time to failure of 4.6 years $(2$ months to 10.4 years). In four patients there was a further injury. In the 25 failures, seven allografts were simply removed and 18 progressed to joint replacement (ten TKRs, eight UKRs).

Using Cox's proportional hazards model, medial versus lateral allografts showed a non-significant hazard $(\mathrm{p}=0.848)$. Kaplan-Meier survival analysis showed mean survival times of 9.9 years (SD $0.5 ; 95 \%$ CI 9.0 to $10.8,1.3$ to 12.3$)$ for medial allograft $(\mathrm{n}=85)$ and 10.2 years (SD 0.8; $95 \%$ CI 8.6 to $11.7,2$ months to 12.3 years) for lateral allografts $(\mathrm{n}=34)$ (Fig. 3).

Cox's proportional hazards model showed that significant factors on the relative odds of allograft failure were the age of 
Table I. Cox's proportional hazards variables

\begin{tabular}{|c|c|c|c|}
\hline Model variances & p-value & Hazard ratio & $\begin{array}{l}95 \% \text { confidence } \\
\text { interval for hazard } \\
\text { ratio }\end{array}$ \\
\hline Patient gender ( $0=$ male; 1 = female $)$ & 0.094 & 2.466 & 0.859 to 7.084 \\
\hline Medial vs lateral allograft ( $0=$ medial; $1=$ lateral $)$ & 0.848 & 1.109 & 0.387 to 3.180 \\
\hline Number of previous operations & $0.006^{*}$ & 1.529 & 1.133 to 2.065 \\
\hline Joint space narrowing & 0.907 & 1.038 & 0.552 to 1.952 \\
\hline Severity of joint malalignment & 0.535 & 1.790 & 0.285 to 11.254 \\
\hline Malalignment type & 0.939 & & \\
\hline Varus vs neutral & 0.902 & 0.864 & 0.084 to 8.859 \\
\hline Valgus vs neutral & 0.916 & 1.127 & 0.121 to 10.535 \\
\hline Number of additional procedures & 0.780 & 0.922 & 0.522 to 1.628 \\
\hline Osteotomy performed concomitantly ( $0=\mathrm{No} ; 1=$ Yes) & 0.272 & 0.444 & 0.105 to 1.888 \\
\hline Number of concomitant procedures & 0.102 & 1.305 & 0.949 to 1.795 \\
\hline Age at time of meniscal allograft & $0.026^{*}$ & 1.061 & 1.007 to 1.117 \\
\hline Intra-operatively determined $\mathrm{OB}^{\dagger}$ grade $(3$ vs 4$)$ & 0.098 & 5.718 & 0.723 to 45.231 \\
\hline Articular paste graft performed concomitantly ( $0=\mathrm{No}, 1=$ Yes $)$ & 0.371 & 1.628 & 0.559 to 4.736 \\
\hline
\end{tabular}

Table II. Description of failures

\begin{tabular}{llllc}
\hline Removal $^{*}$ & Before 2 years & $\mathbf{2}$ to $\mathbf{5}$ years & $\mathbf{5}$ to $\mathbf{1 0 . 5}$ years & Total failures per type \\
\hline Removal & 3 & 2 & 2 & 7 \\
UKR & 2 & 3 & 3 & 8 \\
TKR & 2 & 3 & 5 & 10 \\
Failures per time period (N) & 7 & 8 & 10 & 25 \\
\hline
\end{tabular}

* UKR, unicompartmental knee replacement; TKR, total knee replacement

the patient at the time of allograft $(\mathrm{p}=0.006)$ and the number of previous procedures to the affected knee $(\mathrm{p}=0.026)$. In patients equivalent in all factors except one year of age, the relative risk of failure is 1.061 (95\% CI 1.007 to 1.117 ) times greater for the older patient. Kaplan-Meier estimated survival time for patients aged 50 years and above was 8.8 years (SD 0.7, $95 \%$ CI 7.5 to 10.2 ) and for those under 50 was 10.7 years (SD 0.5, 95\% CI 9.8 to 11.6 ) (Fig. 4). In patients equal in all factors except the number of previous operations, the relative risk of failure is 1.528 (95\% CI 1.133 to 2.065$)$ times greater for the patient undergoing one more previous procedure.

Patient-reported subjective outcomes. The mean IKDC, WOMAC, and Tegner index outcomes are shown in Figures 5 to 8 . Apart from the Tegner index score seven years post-operatively, all patient-reported subjective outcome scores showed a significant improvement at all intervals. Comparison between patient-reported subjective outcome scores over the two to 12 years of follow-up showed no significant changes, indicating maintenance of improvement over time. Wilcoxon's rank-sum revealed a non-significant change of median Tegner index from baseline to seven years $(\mathrm{p}=0.076)$. At baseline, the mean Tegner index score was 0.38 (SD $0.22 ; 0$ to 1 ); the mean Tegner score was 3.2 (SD 2.0; 0 to 9). At seven years, the Tegner index ratio was 0.49 (SD $0.28 ; 0$ to 1 ). The mean Tegner score was 3.9 (SD 2.3; 0 to 7). It should be noted that the comparison of seven-year scores with baseline is based on a small number of reported scores $(n=21)$, and that no significant difference is seen when seven-year Tegner index data are compared with the two- $(\mathrm{p}=0.159)$, three- $(\mathrm{p}=0.159)$, five$(\mathrm{p}=0.170)$ or ten-year $(\mathrm{p}=0.842)$ post-operative intervals, using the Wilcoxon's rank-sum test.

In only seven knees $(5.8 \%)$ was more severe pain described at the most recent follow-up on the isolated WOMAC pain question compared with the baseline value. Median baseline pain score was 1 (mild) among these patients, and all nine reported only one pain level higher at the most recent follow-up.

\section{Discussion}

This is the longest and largest prospective evaluation to date of concurrent meniscal allograft transplantation and articular cartilage repair in patients with significant chondral damage. Previous studies on meniscal allograft transplantation involved patients with minimal chondral damage ${ }^{11,28}$ and described the results before operation and at the most recent follow-up. $5,7,11,12,21,29$ With follow-up ranging from two to 12 years in our study, we chose to 


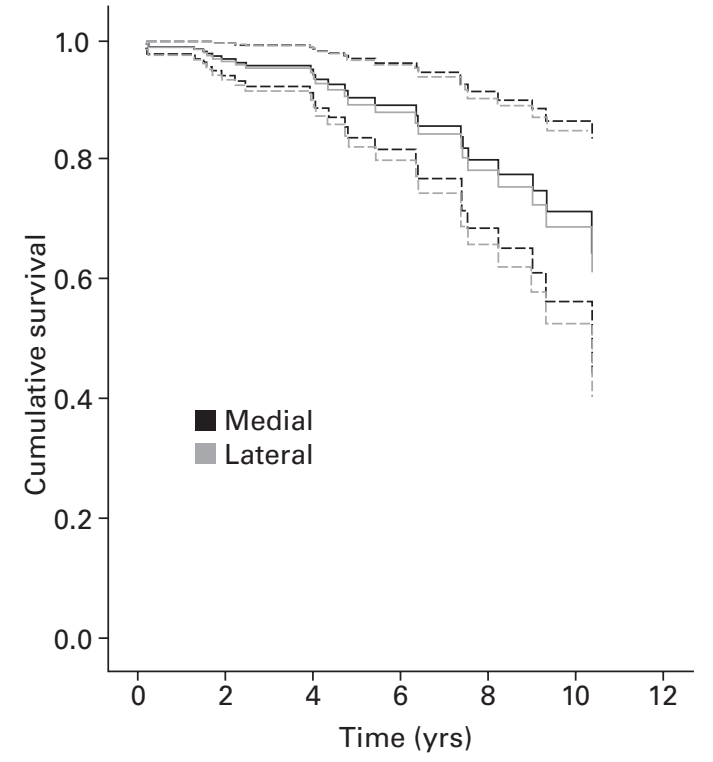

Fig. 3

Graph showing the mean Kaplan-Meier predicted survival time for medial allografts $(n=85) 9.9$ years (SD 0.5), and for lateral allografts $(n=34) 10.2$ (SD 0.8). A non-significant $(p=0.848)$ hazard ratio of 1.109 was observed for lateral cases.

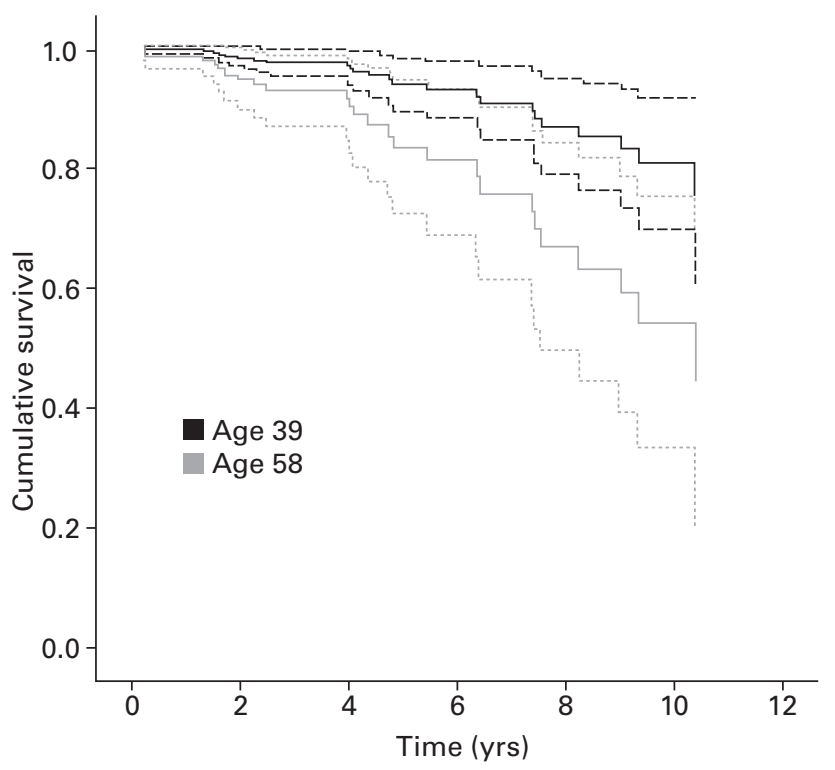

Fig. 4

Graph showing survival distribution of a 39-year-old patient versus 58year-old patient based on a comparison of survival distribution for a patient at the mean age of the group of patients under 50 years old at the time of meniscal allograft transplantation and a patient at the mean age of the group over 50 years old. Kaplan-Meier mean survival time was 8.8 years (SD $0.7 ; 95 \% \mathrm{Cl} 7.5$ to 10.2 ) for the group under 50 years old and 10.67 years (SD $0.5 ; 95 \% \mathrm{Cl} 9.8$ to 11.6 ) for the group over 50 years old. The cumulative hazard ratio is 2.90 .

report patient outcomes at specific time intervals. This gives a more descriptive picture of the patient's experience over time and allows for comparison of patient-reported out-

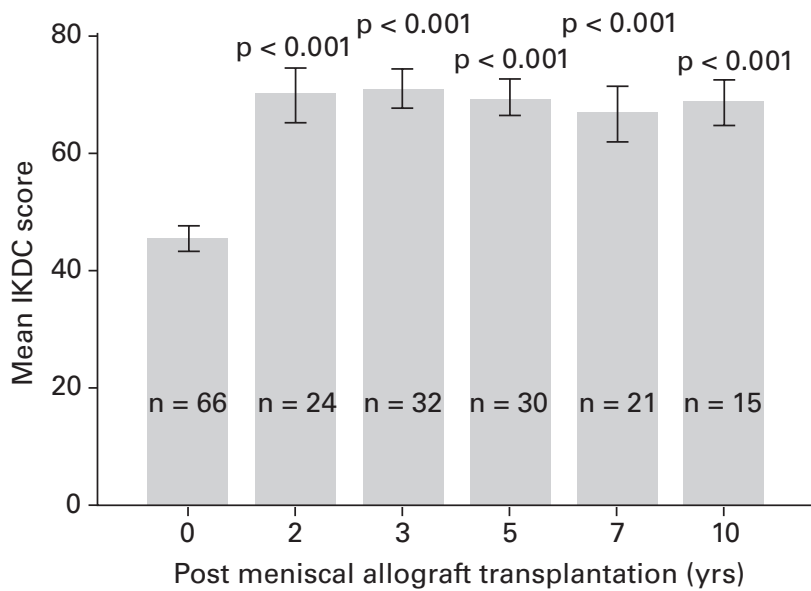

Fig. 5

Graph showing Mean International Knee Documentation Committee (IKDC) scores at baseline and at follow-up. There was a significant improvement in IKDC scores from baseline at all intervals. No significant differences were found among scores during follow-up. The p-values from rank-sum comparison to baseline are shown for all follow-up time intervals. Numbers of assessments for each interval is noted. Error bars represent \pm 1 standard error.

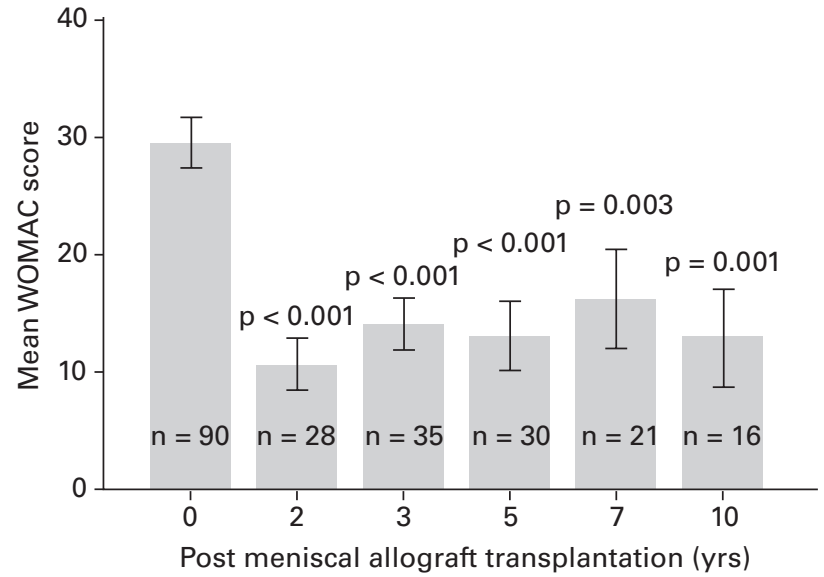

Fig. 6

Graph showing the mean Western Ontario MacMasters Universities Osteoarthritis Index (WOMAC) scores at baseline and at follow-up. There was a significant improvement in WOMAC scores from baseline at all intervals. The $p$-values from rank-sum comparison to baseline are shown for all intervals. The number of assessments for each interval is noted. Error bars represent \pm 1 standard error.

comes at different intervals. Apart from the Tegner index score at seven years post-operatively, patients experienced significant improvements in pain, activity and from baseline measures at all follow-up intervals. These improvements were maintained over time with patients experiencing lasting, stable improvements. At completion of the study we found a $79.9 \%$ success rate, with a KaplanMeier mean estimated survival time of 9.9 years. 


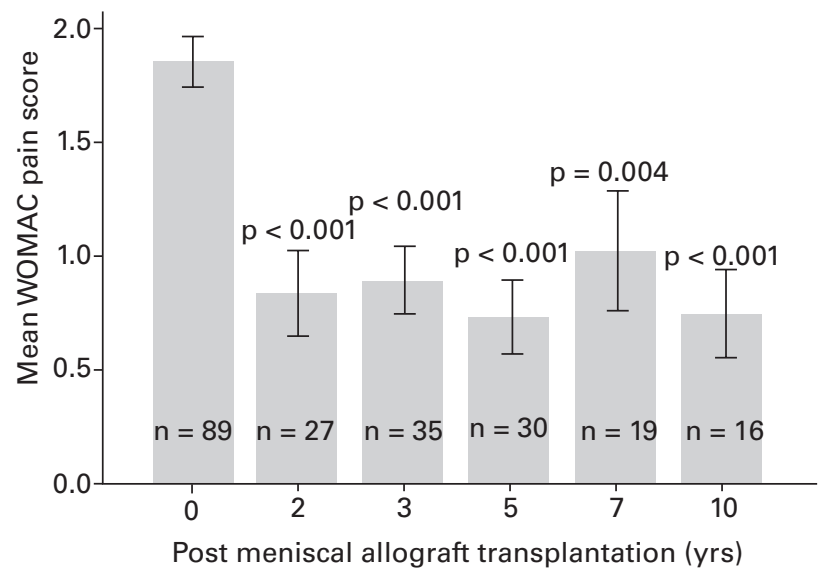

Fig. 7

Graph showing the mean Western Ontario MacMasters Universities Osteoarthritis Index (WOMAC) pain scores at baseline and at follow-up. There was a significant improvement in WOMAC pain scores from baseline at all intervals. The $p$-values from rank-sum comparison to baseline are shown for all intervals. The number of assessments for each interval is noted. Error bars represent \pm 1 standard error.

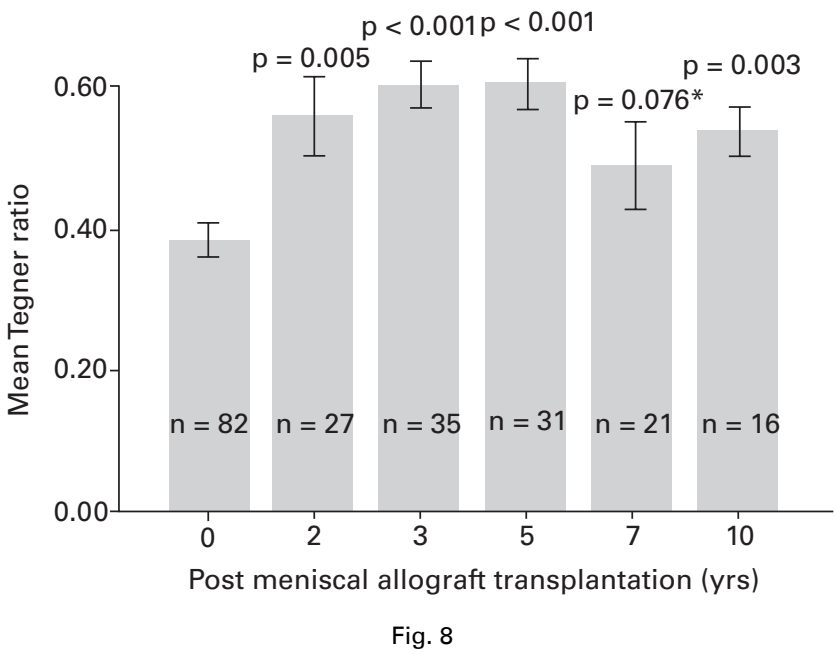

Graph showing the Mean Tegner ratio scores at baseline and at followup. There was a significant improvement in Tegner index scores from baseline at all intervals, except seven years post-operatively $(p=0.076)$. The $p$-values from rank-sum comparison to baseline are shown for all intervals. Number of assessments for each time interval is noted. Error bars represent \pm 1 standard error.

Severe articular cartilage damage is often considered a contraindication for meniscal allograft transplantation. ${ }^{30}$ In all, 97 knees $(81.5 \%)$ in the current study presented with severe damage to the articular cartilage (Outerbridge grade IV); the remaining 22 had Outerbridge grade III changes $(18.5 \%)$. Articular cartilage paste grafting combines an extensive marrow stimulating technique with stem cells, cartilage, and bone grafting for articular cartilage repair. This technique was used in 67 knees $(56.3 \%)$ for severe cartilage damage at the time of allograft transplantation. Other smaller lesions underwent a variety of more tradi- tional techniques of cartilage repair. We believe addressing the damaged articular cartilage at the time of meniscus allograft transplantation is critical to the good long-term results we see in our patients.

The heterogeneity of the patients and the need for combined surgical procedures may have introduced a performance bias into the outcome of the study, making it difficult to identify the influence of the allograft in isolation. ${ }^{31}$ Even so, there was no significant difference in the number of concomitant procedures between those cases that failed and those that did not $(\mathrm{p}=0.333)$. Cole et al ${ }^{11}$ and Noyes et $\mathrm{al}^{28}$ studied heterogeneous patient populations which included those with meniscal allograft transplantation performed in isolation and others who required concomitant cartilage repair. Both studies showed that repair of the articular cartilage had no significant effect on the outcome of the allograft transplantation. Our results confirm these findings, suggesting that articular cartilage repair offers equivalent outcomes independent of the degreee of initial damage.

Subsequent surgical intervention is not always indicative of failure of the procedure; 45 of 56 knees requiring further interventions were classified as non-failures at the completion of the study. Others had also noted the benefit of subsequent operations. Rath et $\mathrm{al}^{29}$ observed improvements in pain and function when patients were treated with partial meniscectomy for tears in the allograft. Noyes et $\mathrm{al}^{28}$ described five non-failure-related subsequent procedures in meniscal allograft patients who were then classified as normal by MRI and clinical evaluation at a mean of 3.3 years' follow-up. Considering revision meniscal allograft surgery as a subsequent surgical intervention rather than failure may be considered a weakness of the study. Nevertheless, six revision patients considered their overall treatment successful at their most recent follow-up.

Our study population showed considerable heterogeneity. In order to address this issue, Cox's proportional hazards model was used to account for a variety of potentially significant effects of different clinically relevant variables. Only age and number of previous procedures showed significant effects. In order to evaluate the hazard of age, a plot illustrating the effect of age on the survival was created (Fig. 4). The mean age of patients above (57 years) and below ( 39 years) 50 years of age at the time of original meniscus allograft surgery was used in the model, whereas all other factors were assumed to be at the population mean values. The resulting plot shows that the upper $95 \%$ confidence limit for a 39-year-old patient extends past the estimated survival distribution of a 57-year-old, indicating a chance that these populations are not entirely different. Additionally, the cumulative survival at 10.3 years for both groups was $75.1 \%$ (95\% CI 60 to 90 ) for 39 years of age and $44.1 \%(95 \%$ CI 19 to 69$)$ for 57 years of age, despite the fact that the hazard ratio indicates that the 57 -year-old is 2.90 times more likely to fail before the 39-year-old. 
In many cases failure of combined meniscal allograft transplantation and articular cartilage repair ultimately leads to TKR, which is known to have limited longevity. ${ }^{32}$ The number of TKRs is predicted to increase to 3.4 million by $2030,{ }^{32}$ with increasing costs. ${ }^{33}$ The 18 patients in this study that progressed to UKR and TKR, did so at a mean of 5.1 years (1.3 to 10.4$)$ after allograft transplanation, when their mean age was 59.3 years (30.9 to 72.0$)$. With increasing costs of primary and revision TKR, the economic impact of delaying primary knee replacement using an outpatient arthroscopic procedure for even five years is of interest.

This long-term follow-up study demonstrates that meniscal allograft transplantation combined with articular cartilage repair provides lasting relief of pain and improved function in patients with severe articular cartilage damage. Axial malalignment did not have an effect on outcome. Although it is known that axial malalignment influences compartmental degeneration, ${ }^{34}$ our findings are dramatically different from that which conventional thinking would predict. It might be that it takes more than the duration of this study for malalignment to produce adverse effects on cartilage. All patients in this study presented with Outerbridge grade III to IV articular cartilage changes, and $46.1 \%$ of the cohort was older than 50 years at the time of surgery, criteria which are generally considered as contraindicated for meniscal allograft transplantation. Meniscal allograft transplantation, when combined with articular cartilage repair, need not be limited to young patients with minimal articular cartilage damage, as demonstrated by the results of this study. Biological joint reconstruction may be an appropriate first step for many people with arthritis of the knee.

\section{Supplementary material}

芦 A further opinion by Professor R. Verdonk and a table showing the rehabilitation programme is available with the electronic version of this article on our website at www.jbjs.org.uk

No benefits in any form have been received or will be received from a commercial party related directly or indirectly to the subject of this article.

\section{References}

1. Zielinks B, Donahue TL. 3D finite element model of meniscectomy: changes in joint contact behavior. J Biomech Eng 2006;128:115-23.

2. Lohmander LS, Englund PM, Dahl LL, Roos EM. The long-term consequence of anterior cruciate ligament and meniscus injuries: osteoarthritis. Am J Sports Med 2007;35:1756-69.

3. Gioe TJ, Novak C, Sinner P, Ma W, Mehle S. Knee arthroplasty in the young patient: survival in a community registry. Clin Orthop 2007;464:83-7.

4. Pennington DW, Swienckowski JJ, Lutes WB, Drake GN. Unicompartmenta knee arthroplasty in patients sixty years of age or younger. J Bone Joint Surg [Am] 2003;85-A:1968-73.

5. Rue JP, Yanke AB, Busam ML, McNickle AG, Cole BJ. Prospective evaluation of concurrent meniscus transplantation and articular cartilage repair: minimum 2-year follow-up. Am J Sports Med 2008:36:1770-8.
6. Stone KR, Walgenbach AW, Freyer A, Turek TJ, Speer DP. Articular cartilage paste grafting to full-thickness articular cartilage knee joint lesions: a 2- to 12-year follow-up. Arthroscopy 2006;22:291-9

7. Farr J, Rawal A, Marberry KM. Concomitant meniscal allograft transplantation and autologous chondrocyte implantation: minimum 2-year follow-up. Am J Sports Med 2007:35:1459-66.

8. Stone KR, Walgenbach AW, Turek TJ, Freyer A, Hill MD. Meniscus allograft survival in patients with moderate to severe unicompartmental arthritis: a 2- to 7-year follow-up. Arthroscopy 2006;22:469-78.

9. Simon TM, Jackson DW. Articular cartilage: injury pathways and treatment options. Sports Med Arthrosc 2006;14:146-54.

10. Sgaglione NA. Biologic approaches to articular cartilage surgery: future trends. Orthop Clin North Am 2005;36:485-95

11. Cole BJ, Denis MG, Lee SJ, et al. Prospective evaluation of allograft meniscus transplantation: a minimum 2-year follow-up. Am J Sports Med 2006;34:919-27.

12. Verdonk PC, Demurie A, Almqvist KF, et al. Transplantation of viable meniscal allograft: survivorship analysis and clinical outcome of one hundred cases. J Bone Joint Surg [Am] 2005;87-A:715-24.

13. Outerbridge RE. The etiology of chondromalacia patellae. J Bone Joint Surg $[\mathrm{Br}]$ 1961;43-B:752-7

14. Kellgren JH, Lawrence JS. Radiological assessment of osteo-arthrosis. Ann Rheum Dis 1957;16:494-502.

15. Stone KR, Freyer A, Turek T, et al. Meniscal sizing based on gender, height, and weight. Arthroscopy 2007;23:503-8.

16. Van Thiel GS, Verma N, Yanke A, et al. Meniscal allograft size can be predicted by height, weight, and gender. Arthroscopy 2009;25:722-7.

17. Stone KR, Walgenbach AW. Meniscal allografting: the three-tunnel technique Arthroscopy 2003;19:426-30.

18. Emery CF, Kiecolt-Glaser JK, Glaser R, Malarkey WB, Frid DJ. Exercise accelerates wound healing among healthy older adults: a preliminary investigation. $J$ Gerontol A Biol Sci Med Sci 2005;60:1432-6.

19. Keylock KT, Vieira VJ, Wallig MA, et al. Exercise accelerates cutaneous wound healing and decreases wound inflammation in aged mice. Am J Physiol Regul Integr Comp Physiol 2008;294:R179-84.

20. Wolff J. The law of bone remodelling. New York: Springer-Verlag, 1986:126.

21. Verdonk PC, Verstraete KL, Almqvist KF, et al. Meniscal allograft transplantation: long-term clinical results with radiological and magnetic resonance imaging correlations. Knee Surg Sports Traumatol Arthrosc 2006;14:694-706.

22. Anderson AF, Irrgang JJ, Kocher MS, Mann BJ, Harrast JJ. The Internationa Knee Documentation Committee Subjective Knee Evaluation Form: normative data. Am J Sports Med 2006;34:128-35.

23. Irrgang JJ, Anderson AF, Boland AL, et al; International Knee Documentation Committee. Responsiveness of the International Knee Documentation Committee Subjective Knee Form. Am J Sports Med 2006;34:1567-73.

24. Bellamy N, Buchanan WW, Goldsmith CH, Campbell J, Stitt LW. Validation study of WOMAC: a health status instrument for measuring clinically important patient relevant outcomes to antirheumatic drug therapy in patients with osteoarthritis of the hip or knee. J Rheumatol 1988;15:1833-40.

25. Tegner Y, Lysholm J. Rating systems in the evaluation of knee ligament injuries. Clin Orthop 1985;198:43-9.

26. Rodkey WG, DeHaven KE, Montgomery WH 3rd, et al. Comparison of the collagen meniscus implant with partial meniscectomy: a prospective randomized trial. $J$ Bone Joint Surg [Am] 2008;90-A:1413-26.

27. Spruance SL, Red JE, Grace M, Samore M. Hazard ratio in clinical trials. Antimicrob Agents Chemother 2004;48:2787-92.

28. Noyes FR, Barber-Westin SD, Rankin M. Meniscal transplantation in symptomatic patients less than fifty years old. J Bone Joint Surg [Am] 2004;86-A:1392-404.

29. Rath E, Richmond JC, Yassir W, Albright JD, Gundogan F. Meniscal allograft transplantation: two- to eight-year results. Am J Sports Med 2001;29:410-14.

30. Noyes FR, Barber-Westin SD. Irradiated meniscus allografts in the human knee: a two to five year follow-up study. Orthop Trans 1995;19:417.

31. Matava MJ. Meniscal allograft transplantation: a systematic review. Clin Orthop 2007:455:142-57

32. Kurtz S, Ong K, Lau E, Mowat F, Halpern M. Projections of primary and revision hip and knee arthroplasty in the United States from 2005 to 2030. J Bone Joint Surg [Am] 2007;89-A:780-5

33. Kurtz SM, Ong KL, Schmier J, et al. Future clinical and economic impact of revision total hip and knee arthroplasty. J Bone Joint Surg [Am] 2007;89-A(Suppl 3):144-51.

34. Cerejo R, Dunlop DD, Cahue S, et al. The influence of alignment on risk of knee osteoarthritis progression according to baseline stage of disease. Arthritis Rheum 2002;46:2632-6. 